\title{
Complete myocardial revascularization and Dor technique in a case of left lung agenesis
}

\author{
Rafael García Fuster, MD, PhD, ${ }^{a}$ José Alfonso Buendía, MD, ${ }^{a}$ Jordi Estornell, MD, ${ }^{b}$ and \\ José Anastasio Montero, MD, PhD, ${ }^{a}$ Valencia, Spain
}

$\mathrm{L}$ ung agenesis is a rare congenital malformation usually associated with other anomalies. In some cases it is compatible with long-term survival and its diagnosis may be delayed until the adulthood. ${ }^{1}$ Its association with a cardiac surgical procedure is extremely infrequent. No previous reports of cardiac operations in adults with lung agenesis have been published with the exception of a mitral valve repair in a 55-year-old man. ${ }^{2}$ The successful repair of ventricular septal defect and patent ductus arteriosus in an infant was reported previously. ${ }^{3}$

\section{Clinical Summary}

A 63-year-old man with an anteroseptal myocardial infarction complicated by progressive heart failure was admitted to our hospital. Left lung agenesis was diagnosed in a routine medical examination 20 years ago. A chest radiograph showed absence of left lung parenchyma and left-sided shift of the mediastinum. Spirometry revealed a moderate obstructive-restrictive dysfunction. Echocardiography detected a huge anteroseptal left ventricular (LV) aneurysm and an ejection fraction (EF) of $20 \%(40 \%$ excluding the aneurysm). Magnetic resonance imaging (MRI) showed absence of the left lung and left main bronchus. An LV aneurysm was found in contact with the lateral chest wall (Figure $1, A$ and $B$ ). The estimated EF was $20 \%$. Angiography confirmed 3-vessel coronary artery disease with an EF of $12 \%$.

Aneurysm excision and complete myocardial revascularization were performed. Mechanical ventilation was temporarily interrupted during median sternotomy. The right lung completely covered the heart. The LV was approached after careful dissection of right pleural and aneurysmal adherences. Cardiopulmonary bypass was established and aneurysmectomy was performed first on the beating heart. The heart was rotated and the ventricular apex was easily exposed, the heart being lifted with sponges and pericardial traction. The dyskinetic segments were excluded and the LV was reshaped with two Fontan sutures without a patch (Figure 2). The LV was closed with a continuous mattress suture of 3-0 polypro-

From the Departments of Cardiac Surgery ${ }^{\mathrm{a}}$ and Radiology, ${ }^{\mathrm{b}}$ Universitary General Hospital of Valencia, Spain.

Received for publication Oct 4, 2005; accepted for publication Oct 26, 2005.

Address for reprints: Rafael García Fuster, MD, C/ Artes Gráficas n ${ }^{\circ}$ 4,esc. izda, pta 3, 46010 Valencia, Spain (E-mail: rgfuster@terra.com).

J Thorac Cardiovasc Surg 2006;131:466-7

$0022-5223 / \$ 32.00$

Copyright $\odot 2006$ by The American Association for Thoracic Surgery

doi:10.1016/j.jtcvs.2005.10.029 pylene on pericardial strips. A second running over-and-over suture ensured a hemostatic closure. After aortic crossclamping, cardiopulmonary bypass was conducted with moderate hypothermia and combined antegrade-retrograde blood cardioplegia.

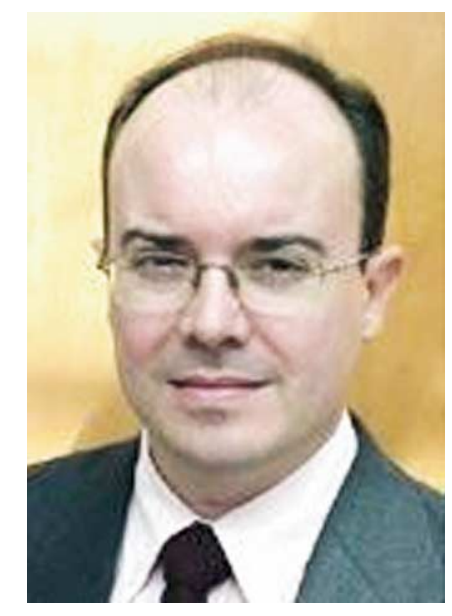

Dr García Fuster Distal anastomoses were performed with saphenous vein on the right and the obtuse marginal coronary arteries. A pedicled left internal thoracic artery was used on the anterior descending artery. Proximal anastomoses were made on the ascending aorta during partial clamping.

The postoperative course was uncomplicated. The patient recovered well and, 3 months after surgery, repeat echocardiography and MRI showed absence of residual aneurysm with recovery of LV dimension and function: estimated EF, 45\% (Figure 1, $C$ and $D$ ). Eighteen months after the operation, the patient is in New York Heart Association class I.

\section{Discussion}

Pulmonary agenesis is a rare congenital anomaly. The incidence of unilateral lung agenesis was estimated at 1 in 10,000 to 15,000 autopsies. ${ }^{4}$ Clinical symptoms usually appear early after birth, and the diagnosis is established during the prenatal or neonatal period in half of all reported cases. Mortality is higher in right lung agenesis and when other malformations are associated. A higher postoperative morbidity and mortality has been observed when the surgical treatment of other anomalies is needed. A case of rightsided congenital diaphragmatic Bochdalek hernia with pulmonary hypoplasia and postoperative massive mediastinal displacement to the right in a neonate was previously reported. ${ }^{5}$ Tracheal compression was the consequence of this severe pulmonary hypoplasia and mediastinal mass effect. Mediastinal stabilization was successfully obtained with an expansion prosthesis in the right hemithorax.

In some patients this disorder is compatible with long-term survival, and most of them remain free of symptoms until a casual diagnosis is made. The usual chest radiographic finding is a marked ipsilateral mediastinal shift and a hypertrophied lung. MRI is the method of choice to accurately define the associated airway and vascular abnormalities.

Median sternotomy may be difficult because the right lung crosses over into the left side of the chest. In our patient a wide exposure was imperative to achieve complete revascularization. A careful dissection of the right pleural reflection into the left side of the chest and over the pericardium was performed. The lung was deflated on cardiopulmonary bypass and the exposure was signif- 


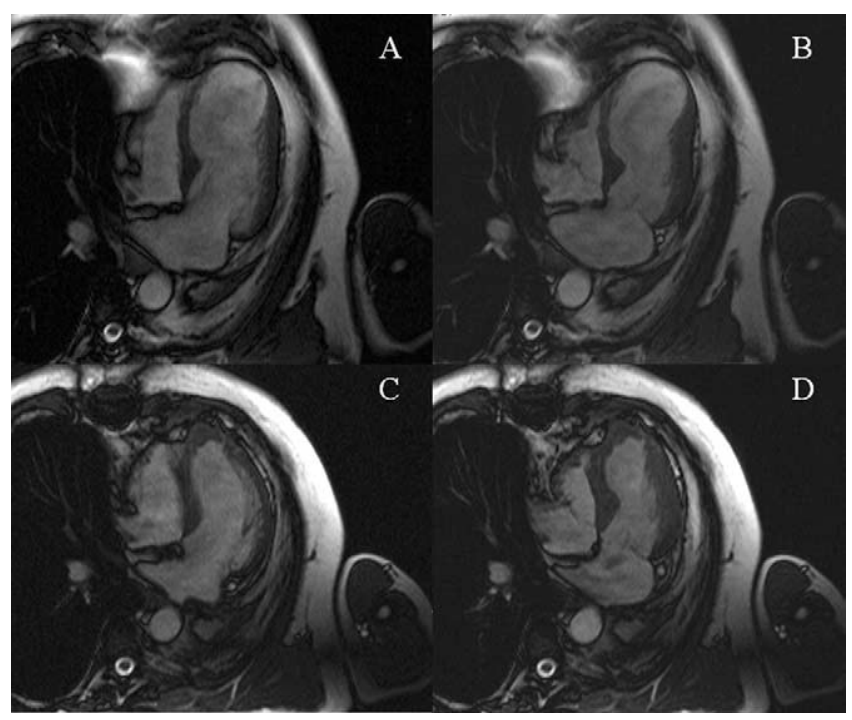

Figure 1. Magnetic resonance images: 4-chamber views (images show the absence of left lung parenchyma). Preoperative left ventricle in diastole (A) and systole (B). Postoperative left ventricle in diastole (C) and systole (D).

icantly improved. After dissection of pericardial adherences over the aneurysm, the rest of the operation were completed.

The interesting aspect of our case is that no previous reports of cardiac operations in adults with lung agenesis have been published with the exception of a mitral valve repair. ${ }^{2}$ Thus, concerns about increased morbidity and mortality in patients undergoing complex cardiac procedures might be raised. This report suggests that complete myocardial revascularization and ventricular aneurysm resection can be performed safely and with excellent results and that left lung agenesis does not pose a special operative risk.

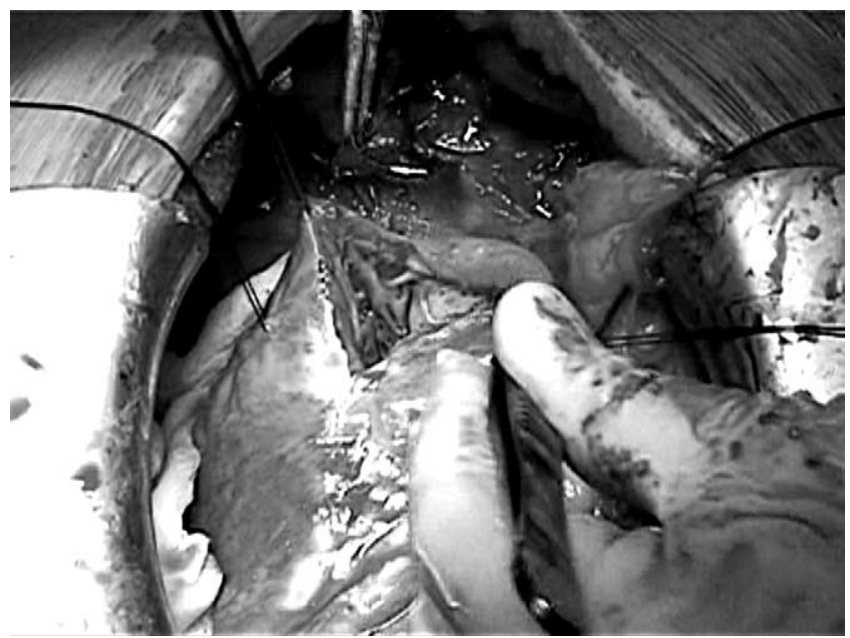

Figure 2. Surgical view showing the left ventricular exposure to perform the Dor technique.

\section{References}

1. Maltz DL, Nadas AS. Agenesis of the lung: presentation of 8 new cases and review of literature. Pediatrics. 1968;42:175-88.

2. Musleh GS, Fernandez P, Jha PK, Hasan R. Mitral valve repair in a 55-year-old man with left lung agenesis. Ann Thorac Surg. 2004;77: 1810-1.

3. Akishima S, Honda M, Kanno M, Shinoka T, Hagino I, Tei I. A successful repair of ventricular septal defect and patent ductus arteriosus associated with pulmonary hypertension in an infant with left lung agenesis. Nihon Kyobu Geka Gakkai Zasshi. 1994;42:2247-51.

4. Olcott CT, Dodey SW. Agenesis of lung in an infant. Am J Dis Child. 1945;65:776-80.

5. Becmeur F, Horta P, Christmann D, Donato L, Livolsi A, de Geeter B, et al. Mediastinal stabilization by an expansion prosthesis in postoperative congenital diaphragmatic hernia with severe pulmonary hypoplasia. Eur J Pediatr Surg. 1995;5:295-8. 